\title{
Über das symmetrische Triaminopyridin
}

Von

Hans Meyer und Erich Ritter v. Beck

Aus dem Chemischen Laboratorium der k. k. Deutschen Universität Prag

(Vorgelegt in der Sitzung am 1. Juli 1915)

Nach der Erschließung von Methoden zur Gewinnung der Diaminopyridine ${ }^{1}$ erschien es verlockend, auch ein Triaminopyridin darzustellen.

Hierfür bot sich in der leicht zugänglichen Citrazinsäure ein geeignetes Ausgangsmaterial.

Wir hofften zunächst, die Dioxyisonicotinsäure im Wege über die Dichlorisonicotinsäure in Diaminopyridincarbonsäure und weiter nach den Abbaumethoden von Hofmann oder von Curtius in Triaminopyridin verwandeln zu können:<smiles>NC1CC(N)CC(N)C1</smiles><smiles>COC(=O)C1CC(N)CC(N)C1</smiles>

1 Hans Meyer mit Mally, Staffen und Tropsch, Monatshefte für Chemie, 33, 393 (1912); 34, 517 (1913); 35, 189, 207 (1914). 
respektive:<smiles>CC(=O)C1CC(N)C(N)CC1C(N)=O</smiles><smiles>CCOC(=O)NC1CC(N)C(N)C(N)C1</smiles>

Die Darstellung der Diaminopyridincarbonsäure ist uns zwar nach mehreren vergeblichen Anläufen gelungen, aber das daraus erhaltene Amid ließ sich nicht mit alkalischer Bromlauge abbauen, und auch das Verfahren von Curtius führte nicht zum Ziel, da es nicht gelang, das Azid:

darzustellen.<smiles>NC1CC(C(=O)N2N=N2)CC(N)N1</smiles>

Um die Wirkung der salpetrigen Säure auf die Amingruppen zu paralysieren, wurden zwei Benzoylreste eingeführt, aber die Haftfestigkeit derselben erwies sich als zu gering. Beim Behandeln des $\alpha \cdot \alpha^{\prime}$-Benzoylaminopyridincarbonsäureesters

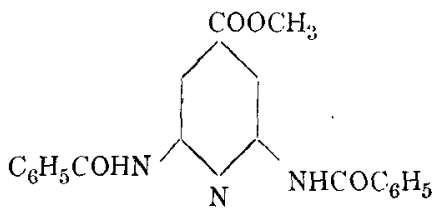

mit Hydrazinhydrat wurde Diaminopyridincarbonsäurehydrazid erhalten. 
Nunmehr wurde versucht, zunächst die Aminogruppe in die $\gamma$-Stellung einzuführen und aus dem so erhaltenen Dichloraminopyridin durch Einwirkenlassen von Ammoniak zum gesuchten Ziele zu gelangen.

Durch den Eintritt der Aminogruppe wird aber das $\boldsymbol{x}$-ständige Halogen, das doch sonst durch so große Labilität ausgezeichnet ist, ganz außerordentlich fixiert, so daß wir zwar eben noch - unter Zuhilfenahme von Kupfer als Katalysator - imstande waren, ein Chlordiaminopyridin

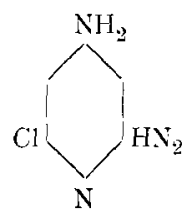

zu gewimnen, aber, in keiner Weise auch das zweite Chloratom zur Reaktion zwingen konnten.

Nachdem diese und manche andere Versuche (so der direkten Einführung von Ammoniak in Citrazinsäure oder ihre Derivate) fehlgeschlagen hatten, haben wir schließlich doch, und zwar in recht einfacher Weise und glatt verlaufender Reaktionsfolge zum Triaminopyridin gelangen können.

Bekanntlich haben Ullmann und Billig ${ }^{1}$ die Halogenatome des 1,4-Dichloranthrachinons durch den Rest des $p$-Toluolsulfamids ersetzen können. Das gleiche Reagens ermöglichte uns die Verwandlung des $\alpha x^{\prime}$-Dichloraminopyridins in Di- $p$-toluolsulfamino- $\gamma$-aminopyridin. Durch konzentrierte Schwefelsäure läßt sich hieraus der Toluolsulfosäurerest leicht wieder abspalten. Das Verseifungsprodukt ist reines Triaminopyridin:

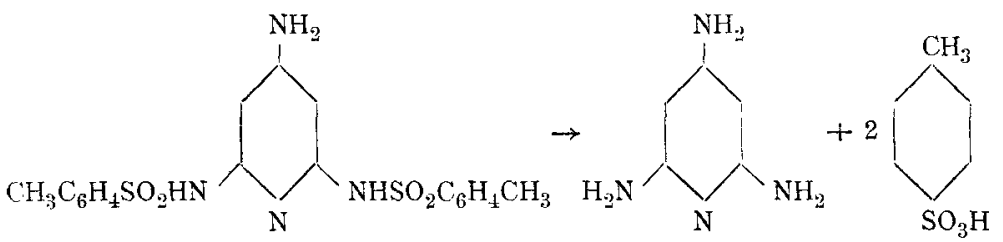

1 Annalen, 381, 17 (1911). 


\section{Experimenteller Teil.}

Die Citrazinsäure erhielten wir in guter Ausbeute nach dem Verfahren von A. Behrmann und A. W. Hofmann als gelbes Pulver, das nur mit Wasser gewaschen zu werden brauchte und schon nahezu reine Säure ergab. Die weitere Reinigung konnte leicht ausgeführt werden durch Lösen in Ammoniak und Wiederausfällen der filtrierten Lösung mit Salzsäure. Doch war auf diesem Weg eine schwache Grünfärbung der Säure nicht zu vermeiden. Die alkalische Lösung der Citrazinsäure war dunkelviolett gefärbt und fluoreszierte sehr stark blau. Rein weiße Säure erhielten wir durch Verseifung des Amids ${ }^{1}$ mit Kalilauge und nachheriges Fällen mit Salzsäure.

\section{Darstellung der $a \alpha^{\prime}$-Dichlorisonicotinsäure.}<smiles>O=C(O)CCCl</smiles>

Besser ais nach der Vorschrift von Behrmann und Hofmann, ${ }^{2}$ die die Chlorierung der Citrazinsäure mit Phosphorpentachlorid und wenig Phosphoroxychlorid durchführten, gelingt die Dichlorisonicotinsäure nach $\mathrm{K}$. Bittner, ${ }^{3}$ der zur Chlorierung lediglich Phosphoroxychlorid verwendet.

Wir führten die Darstellung in der Weise durch, daß wir Citrazinsäure mit der dreifachen Menge Phosphoroxychlorid im Bombenrohr durch 4 Stunden auf etwa $200^{\circ}$ erhitzten. Nach dem Erkalten wurde in Eiswasser gegossen und kurze Zeit bis zum Erstarren der dunkel gefärbten, am Boden lagernden öligen Schicht am Wasserbad erwärmt. Nach dem Erkalten wurde abgesaugt und aus verdünntem Alkohol unter Tierkohlezusatz umkrystallisiert. Farblose Nadeln oder Blättchen. Schmelzpunkt $210^{\circ}$. Ausbeute theoretisch.

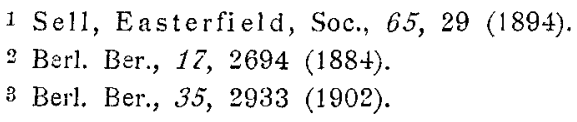


$0 \cdot 1635 g$ Säure ergaben $0.2438 \mathrm{~g} \mathrm{AgCl}$.

In 100 Teilen:

$$
\text { Ch...... } \underbrace{\text { Gefunden }}_{36 \cdot 89} \quad \underbrace{\text { Berechnet }}_{36 \cdot 95}
$$

\section{Der Methylester der Dichlorisonicotinsäure}<smiles>COC(=O)C1CCNC(Cl)C1</smiles>

wurde dargestellt durch Einleiten von trockenem Salzsäuregas in eine Lösung von Dichlorisonicotinsäure in Methylalkohol. Nach dem Absättigen der alkoholischen Lösung mit Salzsäuregas fiel der Ester in schwach gelblich gefärbten, schönen Nadeln aus, die nach mehrmaligem Umkrystallisieren aus verdünntem Alkohol farblos wurden und beim langsamen Erkaltenlassen der sehr verdünnten Alkohollösung eine Länge von mehreren Zentimetern erreichten. Zur Analyse wurde der so gereinigte Ester in wenig starkem Alkohol gelöst und diese Lösung mit Wasser verdünnt. Dabei fiel der Ester als weißer Krystallbrei von ganz kleinen Nadeln aus, die im Vakuum über Schwefelsäure getrocknet wurden. Schmelzpunkt $82^{\circ}$.

Unlöslich in kaltem, wenig löslich in heißem Wasser, löslich in Alkohol, sehr leicht löslich in Äther. $0.0976 \mathrm{~g}$ ergaben $0.1103 \mathrm{~g} \mathrm{AgJ}$.

In 100 Teilen:

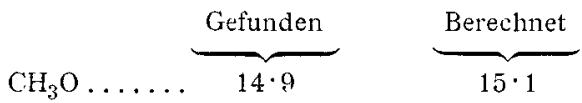

$0 \cdot 1085 \mathrm{~g}$ lieferten $0 \cdot 1503 \mathrm{~g} \mathrm{AgCl}$.

In 100 Teilen:

$$
\mathrm{Cl} \ldots \ldots \ldots \underbrace{\text { Gefunden }}_{34 \cdot 7} \quad \underbrace{\text { Berechnet }}_{34 \cdot 4}
$$

Nun schritten wir an die Darstellung des Hydrazids, indem wir Hydrazinhydrat in kleinem Überschuß auf den 
Methylester einwirken ließen. Die Reaktion ging aber weiter als beabsichtigt, und ein Chloratom wurde unter Ersatz der Hydrazogruppe abgespalten. Es entstand so das

a-Hydrazo- $\alpha-$-chlorpyridin-ך-carbonsäurehydrazid.<smiles>NC(=O)CC(N)N</smiles>

Dichlorisonicotinsäuremethylester wurde mit zirka $15 \%$ Überschuß an Hydrazinhydrat überschichtet. Auf dem Wasserbade trat nach einigen Minuten vollständige Lösung unter Gelbfärbung ein. Nach kurzem Stehnlassen am Wasserbad erstarrte die Flüssigkeit plötzlich $z u$ einem Brei gelblich gefärbter, kleine Büschelchen bildender Nadeln, die nach dem Erkalten abgesaugt und mit Alkohol gewaschen wurden. Das auf diese Weise gewonnene rohe Hydrazid wurde aus sehr stark verdünntem, heißen Alkohol umkrystallisiert und wuchs beim Erkalten in Gestalt kleiner, farbloser Nadeln vom Schmelzpunkt $226^{\circ}$ (unter Rotfärbung) an. Beim Erhitzen etwas über den Schmelzpunkt zersetzte sich das Hydrazid unter Braunfärbung.

Löslich in heißem Wasser, verdünntem Alkohol, Eisessig, schwer löslich in Aceton, unlöslich in Alkohol, Äther, Benzol und Chloroform.

$0.1743 \mathrm{~g}$ ergaben bei $744 \mathrm{~mm}$ und $22^{\circ} 54 \cdot 6 \mathrm{~cm}^{3}$ feuchten Stickstoff.

In 100 Teilen:

$$
\text { N ....... } \underbrace{\text { Gefunden }}_{34 \cdot 5} \quad \underbrace{\text { Berechnet }}_{34 \cdot 7}
$$

Da, wie schon oben bemerkt, bei der Einwirkung von Hydrazinhydrat auf den Methylester der Dichlorisonicotinsäure ohne Anwendung eines Lösungsmittels das eine der beiden $\boldsymbol{\alpha}$-ständigen Chloratome durch die Hydrazogruppe ersetzt wurde, was nicht im Sinn der Abbaufolge zum Dichloraminopyridin lag, wurde versucht, diesem Übelstand 
vorzubeugen. Dies gelang auch, als die Einwirkung von Hydrazinhydrat auf den Methylester in einem geeigneten Lösungsmittel - Äthylalkohol - vorgenommen wurde.

\section{๙. $x^{\prime}$-Dichlorisonicotinsäurehydrazid.}<smiles>NC(=O)CCCl</smiles>

Der Methylester der Dichlorisonicotinsäure wurde in der Kälte in Äthylalkohol gelöst und die Lösung mit etwas mehr als der berechneten Menge Hydrazinhydrat (zirka 15\% Überschuß) versetzt. Selbst nach fünfstündigem Kochen am Rückflußkühler schied sich das Hydrazid nicht ab. Der Kolbeninhalt wurde nun auf die Hälfte eingedampft, worauf beim Erkalten das Hydrazid als schwach gelb gefärbter Krystallbrei von kleinen Nädelchen sich abschied. Aus verdünntem Alkohol erhielt man es in glänzend weißen Nadeln vom Schmelzpunkt $184^{\circ}$.

$0.0631 \mathrm{~g}$ lieferten bei $745 \mathrm{~mm}$ und $23^{\circ} 11.0 \mathrm{~cm}^{3}$ feuchten Stickstoff.

In 100 Teilen:

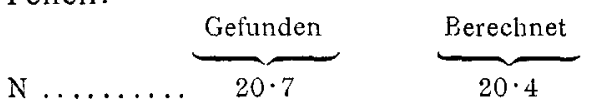

Löslich in den gebräuchlichsten Lösungsmitteln, wie Alkohol, Aceton, Eisessig; in Wasser, Äther, Chloroform, Benzol und Xylol erst beim Erwärmen.

$\alpha \alpha^{\prime}$-Dichlorisonicotinsäureazid.<smiles></smiles>

$5 g$ Dichlorisonicotinsäurehydrazid wurden in einem Becherglas mit $400 \mathrm{~cm}^{3}$ Wasser überschüttet und $30 \mathrm{~cm}^{3}$ 
2 normaler Salzsäure dazugegeben. Nach einiger Zeit und mehrmaligem Durchschütteln trat klare Lösung ein. Das Becherglas wurde nun in Eis gekühlt und $3.5 \mathrm{~g}$ Natriumnitrit in konzentrierter wässeriger Lösung unter Umrühren zufließen gelassen. Das Azid schied sich als weißer Schaum auf der Flüssigkeit ab, wurde abgesaugt, mit Wasser gewaschen und im Vakuumexsikkator über Schwefelsäure getrocknet. Schmelzpunkt $89^{\circ}$.

Das Azid verpufft nur schwach auf dem Platinspatel, im Gegensatz zu andern Aziden der Pyridinreihe. Es reizt die Nasenschleimhäute ziemlich stark.

\section{a. $x^{\prime}$-Dichlorisonicotinsäureurethan.}<smiles>CCOC(=O)NC1CCC(Cl)C1</smiles>

Dichlorisonicotinsäureazid wurde mit der zwanzigfachen Menge absoluten Alkohols unter Feuchtigkeitsabschluß 2 Stunden am Rückflußkühler auf dem Wasserbade erhitzt, dann der überschüssige Alkohol abdestilliert und das Urethan im Vakuum über Schwefelsäure zum Auskrystallisieren gebracht. Aus sehr stark verdünntem Alkohol, von dem ein großer Überschuß genommen werden mußte, erhielt man es in schönen farblosen Nadeln vom Schmelzpunkt $132^{\circ}$. Wird durch Reiben elektrisch.

Löslich in Alkohol und den gebräuchlichsten Lösungsmitteln, wie Äther, Aceton, Chloroform, Eisessig (Benzol beim Erwärmen), unlöslich in kaltem, schwer löslich in heißem Wasser.

Die Äthoxylbestimmung war in normaler Zeit beendigt. $0.1076 \mathrm{~g}$ lieferten $0.1059 \mathrm{~g} \mathrm{AgJ}$.

In 100 Teilen:

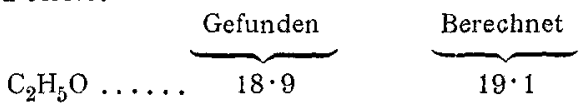




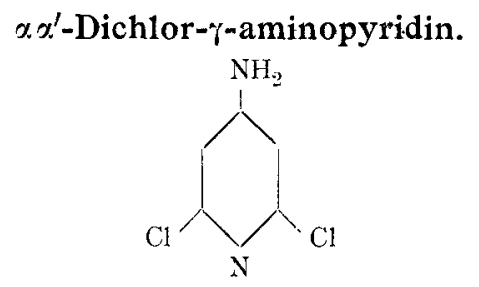

Die Verseifung des Dichlorisonicotinsäureurethans zum Dichloraminopyridin gelang zwar mit alkoholischem Kali, das bei der Darstellung des $\alpha \cdot \alpha^{\prime}$-Diaminopyridins sich als geeignet erwiesen hatte, ${ }^{1}$ doch waren die Ausbeuten unbefriedigend. Erst als die Verseifung des Urethans mit wässerigalkoholischer Lauge durchgeführt wurde, war das Ergebnis zufriedenstellend und die Ausbeute nahezu quantitativ.

$24 \mathrm{~g}$ Dichlorisonicotinsäureurethan wurde in nicht $\mathrm{zu}$ viel heißem Alkohol gelöst, mit einer Lösung von $14 \mathrm{~g} \mathrm{KOH}$ in Wasser versetzt und am Rückflußkühler in lebhaftem Sieden erhalten. Da beim Versetzen der alkoholischen Lösung des Urethans mit Kalilauge das Urethan teilweise wieder ausgefallen war, wurde während des Siedens Alkohol bis zur klaren Lösung nachgegossen. Nach dreistündigem Erhitzen am Rückfluß war die Verseifung beendet.

Die Flüssigkeit hatte, über Nacht stehn gelassen, das

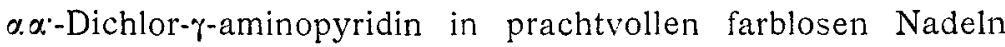
abgeschieden, die nach dem Absaugen und Umkrystallisieren aus verdünntem Alkohol konstant bei $176^{\circ}$ schmolzen.

Das alkalische Filtrat wurde zur Trockene eingedampft, auf Ton abgepreßt und mehrmals mit trockenem Benzol extrahiert. So konnten die restlichen Mengen des Dichloraminopyridins gewonnen werden. Ausbeute $15 \mathrm{~g}$.

Sublimiert unzersetzt. Zeigt schwache Carbylaminreaktion. Fehling'sche Lösung und ammoniakalische Silbernitratlösung werden auch in der Wärme nicht reduziert.

Löslich in Alkohol, Äther, Benzol, Aceton (Eisessig und Chloroform erst beim Erwärmen), unlöslich in kaltem, löslich in heißem Wasser, unlöslich in kaltem, löslich in heißem Ammoniak.

1 H. Meyer und J. Mally, Monatshefte für Chemie, 33, 393 (1912). 
I. $0.2038 \mathrm{~g}$ ergaben bei $740 \mathrm{~mm}$ und $22^{\circ} 31 \cdot 7 \mathrm{~cm}^{3}$ feuchten Stickstoff.

II. $0 \cdot 1477 g$ gaben, mit $\mathrm{CuO}$ und gekörntem $\mathrm{PbCrO}_{4}$ verbrannt, $0 \cdot 1982 g$ $\mathrm{CO}_{2}$ und $0.0323 \mathrm{~g} \mathrm{H}_{2} \mathrm{O}$.

In 100 Teilen:

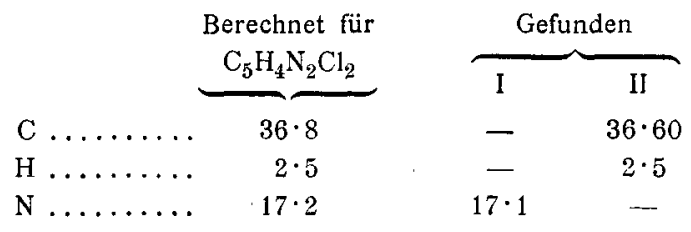

$\alpha-$ Amino- $\alpha$ '-chlorpyridin- $\gamma$-carbonsäure.<smiles>NC1CN=NC1</smiles>

Dichlorisonicotinsäure ${ }^{1}$ wurde mit überschüssigem konzentrierten Ammoniak 8 Stunden im Autoklaven auf ungefähr $200^{\circ}$ erhitzt. Nach dem Erkalten wurde die klare, gelblich gefärbte Lösung mit verdünnter Salzsäure im Überschuß versetzt. Dabei ging die Aminochlorpyridincarbonsäure, die in der neutralen und schwach sauren Lösung ausgefallen war, wieder in Lösung und etwas unveränderte Dichlorisonicotinsäure blieb ungelöst zurück. Nach dem Filtrieren wurde die saure Flüssigkeitsmenge mit Ammoniak bis zur schwach sauren Reaktion abgestumpft, wobei die Aminochlorpyridincarbonsäure in gelben kleinen Nadeln erbalten werden konnte. Sie wurde aus heißem Wasser umkrystallisiert und schmilzt auf dem Platinspatel bei hoher Temperatur unter Zersetzung.

I. $0.0851 \mathrm{~g}$ lieferten $0.0693 \mathrm{~g} \mathrm{Ag} \mathrm{Cl}$.

II. $0 \cdot 1954 \mathrm{~g}$ gaben bei $730 \mathrm{~mm}$ und $24^{\circ} 30 \cdot 4 \mathrm{~cm}^{3}$ feuchten Stickstoff.

In 100 Teilen:

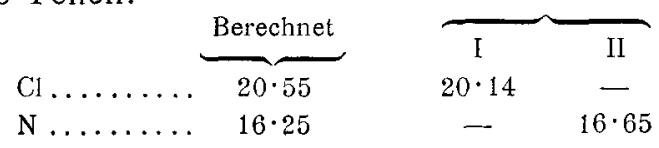

1 Sel1, Dootson, Soc., 71, 1075 (1897). 


\section{$\alpha . x^{\prime}$-Diaminopyridin- $\gamma$-carbonsäure.}<smiles>NC1CC(N)CC(N)C1</smiles>

Bei sechs- bis achtstündigem Erhitzen von Dichloris Jnicotinsäure mit überschüssigem konzentrierten Ammoniak (bei $0^{\circ}$ gesättigt) auf $210^{\circ}$ im Autoklaven unter Zusat $z$ von etwas Kupferbronze konnte der Ersatz beider Chloratome durch die Aminogruppe erreicht werden. Nach dem Erkalten wurde der Autoklaveninhalt ron der Kupferbronze abfiltriert und mit überschüssiger verdünnter Salzsäure behandelt, wobei die Diaminopyridincarbonsäure in Lösung ging, während wenig unveränderte Dichlorisonicotinsäure ungelöst blieb. Nach dem Filtrieren und Abstumpfen des Filtrats mit verdünntem Ammoniak bis zur schwach sauren Reaktion fiel die Säure als gelbes Pulver aus, das in allen gebräuchlichen Lösungsmitteln unlöslich war. Durch wiederholtes Lösen in Ammoniak und Wiederausfällen mit Salzsäure konnte eine annähernd reine Säure erhalten werden.

Am Platinspatel verkohlte die Säure, ohne zu schmelzen, während die Aminochlorpyridincarbonsäure, wie schon früher erwähnt, bei hoher Temperatur unter Zersetzung schmolz.

Durch Verseifen des weiter unten beschriebnen Amids mit Kalilauge und vorsichtiges Ansäuern mit Salzsäure bis zur schwach sauren Reaktion erhielt man die Säure in schönen farblosen Nadein. Die Versuche, diese Säure durch Einwirkung von Ammoniak auf Citrazinsäure zu erhalten, führten nicht zum Ziel.

Stickstoffbestimmung in der rohen Säure:

$0.1179 \mathrm{~g}$ ergaben bei $752 \mathrm{~mm}$ und $24^{\circ} 28.2 \mathrm{~cm}^{3}$ feuchten Stickstoff.

In 100 Teilen:

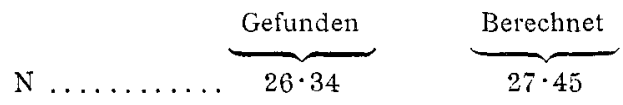




\section{$\alpha . \alpha^{\prime}$-Diaminopyridin- $\gamma$-carbonsäuremethylester.}<smiles>CC(=O)CC1CN=C(N)C(N)C1</smiles>

$\alpha \cdot \alpha^{\prime}$-Diaminopyridincarbonsäure wurde in Methylalkohol suspendiert und trockenes Salzsäuregas eingeleitet. Nach längerm Einleiten trat vollständige Lösung ein. Beim Sättigen dieser alkoholischen Lösung mit Salzsäuregas setzte sich $\alpha \alpha^{\prime}$-Diaminopyridin - $\gamma$-carbonsäuremethylesterdichlorhydrat in grünlichgelb gefärbten Nadeln vom Schmelzpunkt $208^{\circ}$ (unter Zersetzung) ab. Es wurde möglichst gut abgesaugt und ließ sich nur sehr schwer unzersetzt umkrystallisieren. Deshalb wurde von einer weitern Reinigung abgesehen. Die Chlorbestimmung ergab, wie zu erwarten war, einen etwas $z u$ hohen Wert.

$0.2873 \mathrm{~g}$ Chlorhydrat ergaben $0.3521 \mathrm{~g} \mathrm{AgCl}$.

In 100 Teilen:

$$
\text { C1 ........ } \underbrace{\text { Gefunden }}_{30 \cdot 32} \quad \underbrace{\text { Berechnet }}_{29 \cdot 79}
$$

Das rohe Dichlorhydrat wurde mit Natriumbicarbonat in einer Reibschale zerrieben und mit Benzol am Rückflußkühler extrahiert. Nach dem Filtrieren und Abdestillieren des überschüssigen Benzols verblieb der Ester in seidenglänzenden Nadeln, die einen Stich ins Gelbliche zeigten und sich zu einem Filz zusammenlegten. Aus Benzol umkrystallisiert, schmilzt er bei $173^{\circ}$. Wird durch Reiben elektrisch.

Schon in der Kälte sehr leicht löslich in Aceton, schwerer in Methyl- und Äthylalkohol, beim Erwärmen in Benzol, Wasser und Chloroform, fast unlöslich in Äther. Die Lösungen des Esters zeigen blaue Fluoreszenz.

$0.1175 \mathrm{~g}$ ergaben bei der Methoxylbestimmung $0.1649 \mathrm{~g} \mathrm{AgJ}$.

In 100 Teilen:

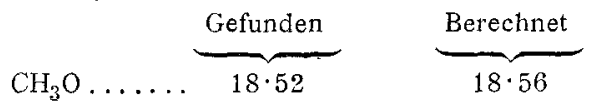


$x \alpha^{\prime}$-Diaminopyridin- $\gamma$-carbonsäureamid.<smiles>NC(=O)C1CC(N)C(N)CC1N</smiles>

Beim Übergießen von Diaminopyridincarbonsäuremethylester (zirka 1 Gewichtsteil) mit dem stärksten, etwa 36 prozentigen Ammoniak des Handels (4 bis 5 Gewichtsteile) und längerm Stehnlassen, wobei wiederholt geschüttelt wurde, schied sich das Amid in schwach gelb gefärbten, rechteckigen Blättchen ab, die aus heißem Wasser in schönen farblosen Blättchen auskrystallisierten und vierstrahlige Sternchen bildeten. Aus der ammoniakalischen Mutterlauge konnten durch freiwilliges Verdunsten derselben noch weitere Mengen des Amids gewonnen werden. Schmelzpunkt $256^{\circ}$ unter Braunfärbung.

$0 \cdot 1321 \mathrm{~g}$ ergaben bei $735 \mathrm{~m} 1 \mathrm{n}$ und $23^{\circ} 44.0 \mathrm{~cm}^{3}$ feuchten Stickstoff.

In 100 Teilen:

$\mathrm{N} \ldots \ldots \ldots \ldots \underbrace{\text { Gefunden }}_{36 \cdot 83} \quad \underbrace{\text { Berechnet }}_{36 \cdot 84}$

Bei dem Versuch, das Amid nach Hofmann abzubauen, entstand eine bromhaltige Substanz, die noch näher untersucht werden wird. Die weitern Versuche mußten vorläufig wegen Mangels an Material bis zu einem geeigneten Zeitpunkt zurückgestellt werden.

$\alpha \alpha^{\prime}$-Diaminopyridin- $\gamma$-carbonsäurehydrazid.<smiles>NNC(=O)C1CC(N)=NC(N)C1</smiles>

Der Methylester der Diaminopyridincarbonsäure wurde in alkoholischer Lösung mit einem ungefähr 15 prozentigen 
Überschuß an Hydrazinhydrat versetzt. Nach halbstündigem Kochen am Rückflußsühler wurde auf die Hälfte eingedampft, wobei die Flüssigkeit zu einem Brei gelb gefärbter, kleiner Nädelchen erstarrte. Aus stark verdünntem Alkohol krystallisierten schöne, zu Büscheln verwachsene Nadeln aus, die sich durch den Schmelzpunkt als unveränderter Methylester herausstellten.

Nun wurde der Methylester direkt mit Hydrazinhydrat behandelt, wobei schon in der Kälte, besser beim Erwärmen am Wasserbad die Umsetzung in das Hydrazid glatt vor sich ging. Die kleinen, farblosen, rechteckigen Blättchen wurden einmal aus verdünntem Alkohol umkrystallisiert und bildeten dann schöne farblose Nadeln, die im offenen Schmelzpunktsröhrchen bei $260^{\circ}$ unter Dunkelrotfärbung schmolzen und sublimierten, während im beiderseits zugeschmolzenen Röhrchen der Schmelzpunkt unter Orangefärbung bei $268^{\circ}$ lag.

$0.1691 \mathrm{~g}$ ergaben bei $744 \mathrm{~mm}$ und $22^{\circ} 63.8 \mathrm{~cm}^{3}$ feuchten Stickstoff.

In 100 Teilen:

$$
\mathrm{N} \ldots \ldots \ldots \ldots \underbrace{\text { Gefunden }}_{41 \cdot 61} \quad \underbrace{\text { Bereclnet }}_{41 \cdot 91}
$$

Die Versuche, mittels der Curtius'schen Abbaufolge von der $\boldsymbol{x} \boldsymbol{\alpha}^{\prime}$-Diaminoisonicotinsäure auf direktem Weg zum entsprechenden $\alpha \gamma \alpha^{\prime}$-Triaminopyridin zu gelangen, mußten aufgegeben werden, weil es nicht möglich war, das Hydrazid in das Azid überzuführen; die salpetrige Säure machte immer ihren zerstörenden Einfluß auf die beiden $\alpha$-ständigen Aminogruppen geltend, wahrscheinlich unter Diazotierung derselben.

Nun wurde noch der Versuch gemacht, durch eine Benzoylierung der beiden Aminogruppen des Methylesters einer Diazotierung derselben durch die salpetrige Säure vorzubeugen. 


\section{Benzoylderivat \\ des Methylesters der Diaminopyridincarbonsäure.}

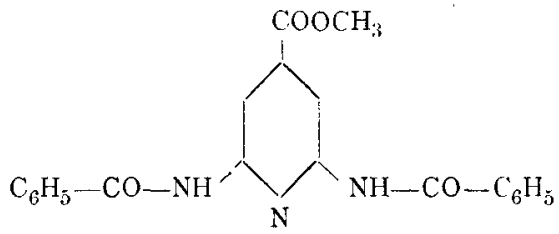

Der Methylester der $\alpha \cdot \alpha^{\prime}$-Diaminopyridin- $\gamma$-carbonsäure wurde mit überschüssigem Benzoylchlorid eine Stunde am Rückflußkühler gekocht, die Reaktionsflüssigkeit in Methylalkohol eingetragen, das abgeschiedene Benzoylderivat abgesaugt und mit Methylalkohol gewaschen. Nach dem weitern Auskochen mit Methylalkohol, um Spuren von Benzoylchlorid und etwa gebildete Benzoesäure zu entfernen, verblieb der benzoylierte Methylester, der auch in heißem Alkohol unlöslich war, in Form kleiner weißer Nädelchen, die bei $120^{\circ}$ getrocknet wurden. Schmelzpunkt im beiderseits zugeschmolzenen Röhrchen $312^{\circ}$.

Sehr schwer löslich in den gebräuchlichsten Lösungsmitteln. Wird durch Reiben sehr stark elektrisch.

$0 \cdot 1077 \mathrm{~g}$ ergaben bei $735 \mathrm{~mm}$ und $28^{\circ} 10 \cdot 9 \mathrm{~cm}^{3}$ feuchten Stickstoff.

In 100 Teilen:

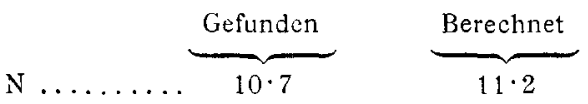

Der benzoylierte Methylester wurde mit möglichst wenig Alkohol zu einem Krystallbrei angerührt und mit Hydrazinhydrat in geringem Überschuß in der bekannten Weise behande!t, um das Hydrazid darzustellen, doch trat dabei eine Abspaltung der beiden Benzoylgruppen ein unter Bildung von $\alpha \alpha^{\prime}$-Diaminopyridin- $\gamma$-carbonsäurehydrazid, wie der Schmelzpunkt und der Mischungsschmelzpunkt mit dem direkt aus dem Methylester der Diaminoisonicotinsäure gewonnenen Hydrazid unzweideutig bewiesen. 
Nach dem Fehlschlagen der Versuche, die Diaminopyridincarbonsäure direkt nach Curtius abzubauen, nahmen wir die Versuche wieder auf, um durch die Einwirkung von konzentriertem Ammoniak, das bei der Amidierung der Dichlorisonicotinsäure so wertvolle Dienste geleistet hatte, auf das Dichloraminopyridin einen Ersatz der beiden Chloratome durch Aminogruppen zu erreichen.

Dichloraminopyridin wurde mit konzentriertem Ammoniak einmal ohne, das anderemal mit einem geringen Zusatz von Kupferbronze, die schon bei der Darstellung der Diaminopyridincarbonsäure aus Dichlorisonicotinsäure auch in der Pyridinreihe ihre gute Wirkung als Katalysator gezeigt hatte, im Bombenrohr durch 4 Stunden auf 180 bis $190^{\circ}$ erhitzt. Beim Öffnen der beiden Bombenröhren zeigte sich, daß in dem Rohr ohne Kupferzusatz keine Reaktion eingetreten war. Der Inhalt erwies sich als unverändertes Dichloraminopyridin, das schon in dem starken Ammoniak im Rohr in Gestalt schöner farbloser Nadeln auskrystallisiert war. Es trat also bei der Anwendung von Ammoniak allein kein Ersatz der Chloratome durch $\mathrm{NH}_{2}$ ein.

Im zweiten Rohr war eine Reaktion vor sich gegangen. Der Inhalt hatte dunkelbraune Farbe angenommen und wurde nach dem Filtrieren zwecks Darstellung eines nicht flüchtigen Chlorhydrates mit verdünnter Salzsäure angesäuert, wobei Farbenumschiag von Braun in Gelb auftrat. Spuren von in Lösung gegangenem Kupfer entfernte man nun durch Einleiten von Schwefelwasserstoff und dampfte das Filtrat zur Trockene ein. Das trockene Chlorhydrat wurde mit Soda innig gemischt und ziemlich oft mit Äther extrahiert. Wir erhielten so eine Substanz, die schwer rein zu isolieren war und nach der Analyse wahrscheinlich ein Diaminochlorpyridin sein dürfte.

Trotzdem diese Versuche ziemlich viel Material gekostet hatten, gelang es nicht, auf diesem Weg aus dem Dichloraminopyridin durch Einwirkung von Ammoniak das Triaminopyridin darzustellen.

Dagegen waren die Amidierungsversuche bei der Anwendung von Paratoluolsulfamid, wie schon erwähnt, von 
Erfolg begleitet. Es gelang so, bei Anwesenheit von Kupfer, beide Chloratome durch den Paratoluolsulfaminrest $z u$ ersetzen und dadurch ein Derivat des Triaminopyridins zu erhalten.

\section{Diparatoluolsulfamino-r-aminopyridin.}

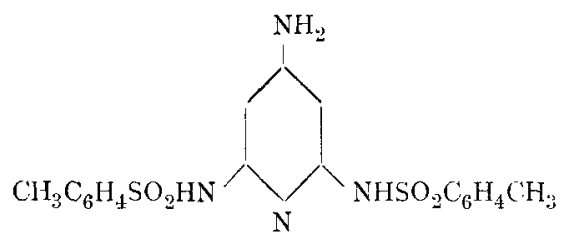

$1 g$ Dichloraminopyridin wurde mit $1 \cdot 3 g$ Paratoluolsulfamid und $0.7 \mathrm{~g}$ reiner, calcinierter Soda unter Zusatz von etwas Kupferbronze in einer kleinen Reibschale innig gemischt und hierauf in einem kleinen Kölbchen im Ölbad durch 4 Stunden auf 180 bis $190^{\circ}$ erhitzt. Dabei sublimierte etwas unverändert gebliebenes Paratoluolsulfamid und blieb in Gestalt schöner Nadeln an den Wänden des Kölbchens haften.

Der Kölbcheninhalt wurde mit heißem, alkohol- und acetonhaltigem Wasser ausgelaugt und vom Kupfer abfiltriert. Beim Erkalten schied sich eine in feinen Nadeln krystallisierende Masse $a b$, die der Hauptmenge nach aus $\alpha \cdot \alpha^{\prime}$-Diparatoluolsulfamino- $\gamma$-aminopyridin und $\mathrm{zu}$ geringen Teilen aus Paratoluolsulfamid bestand.

Nach dem Fiitrieren und Waschen des Rückstandes mit kaltem Wasser wurde auf Ton abgepreßt und im Wassertrockenschrank getrocknet. Durch mehrmaliges Auskochen des vollständig trockenen Rückstandes mit Benzol, in welchem das richt in Reaktion getretene Paratoluolsulfamid löslich ist, konnten die beiden Substanzen ohne allzu große Schwierigkeiten getrennt werden. Das in Benzol unlösliche $\alpha \alpha^{\prime}$-Diparatoluolsulfamin- $\gamma$-aminopyridin wurde einigemale aus sehr stark verdünntem Alkohol unter Tierkohlezusatz umkrystallisiert und auf diese Weise in farblosen, kleinen, glänzenden Nadein erhalten, die bei $360^{\circ}$ noch nicht schmolzen und sich bei höherer Temperatur zersetzten.

Aus dem ersten Filtrate konnten kleine Mengen an unverändert gebliebenem Dichloraminopyridin durch Einengen 
am Wasserbad und Umkrystallisieren aus verdünntem Alkohol gewonnen werden.

$1.475 \mathrm{mg}$ ergaben bei $732 \mathrm{~mm}$ und $21^{\circ} 0.171 \mathrm{~cm}^{3}$ Stickstoff. 1

In 100 Teilen:

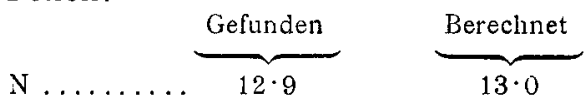

$x_{\gamma} \alpha^{\prime}$-Triaminopyridin.<smiles>NC1CNCCN1</smiles>

Die Verseifung des $\alpha \alpha^{\prime}$-Diparatoluolsulfamino- $\gamma$-aminopyridins zum $x \gamma \alpha^{\prime}$-Triaminopyridin gelang am besten mit konzentrierter Schwefelsäure.

$\mathrm{Zu}$ diesem $\mathrm{Zweck}$ wurde $\alpha \alpha^{\prime}$-Diparatoluolsulfamino$\gamma$-aminopyridin $(1 / 2 g)$ mit konzentrierter Schwefelsäure $\left(2 \mathrm{~cm}^{3}\right)$ 2 Stunden in einem kleinen Kölbchen am Wasserbad erhitzt. Nach dem Erkalten wurde der Kölbcheninhalt in Wasser $\left(50 \mathrm{~cm}^{3}\right)$ gegossen und die überschüssige Schwefelsäure mit Bariumhydroxyd $(11 \mathrm{~g})$ entfernt. Vom abgeschiedenen Bariumsulfat wurde abfiltriert und auf ein Drittel der Flüssigkeitsmenge am Wasserbad eingedampft. Es hatte sich wenig Bariumcarbonat abgeschieden, von welchem neuerdings abfiltriert wurde. Nun dampfte man das Filtrat zur Trockene ein.

Der vollständig trockene Rückstand wurde fein zerrieben, mehrmals mit trockenem Benzol extrahiert und rom paratoluolsulfosauren Barium abfiltriert. Aus dem Benzol schird sich beim Einengen der Lösung am elektrischen Lichtbad und Entfernen des noch vorhandenen Benzols im Vakuum über fester Kalilauge eine in schönen weißen Nädelchen krystallisierende Substanz ab - das $a . \gamma \alpha^{\prime}$-Triaminopyridin. Dasselbe wurde mehrmals aus Toluol umkrystallisiert und zeigte den Schmeizpunkt $185^{\circ}$.

1 Mikroanalyse nach Pregl. 
Es sublimiert unzersetzt, ist hygroskopisch und zeigt schwache Carbylaminreaktion.

Fehling'sche Lösung wird nicht, ammoniakalische Silbernitratlösung erst in der Hitze reduziert. Die wässerige Lösung zeigt stark alkalische Reaktion.

Leicht löslich in Wasser, Alkohol; in Benzol, Aceton, Toluol erst beim Erwärmen, ebenso in Äther.

$1.901 \mathrm{mg}$ Substanz ergaben bei $738 \mathrm{~mm}$ und $19^{\circ} 0.752 \mathrm{~cm}^{3}$ Stickstoff.

In 100 Teilen:

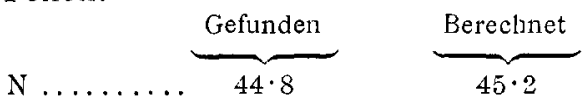

Beim Versetzen einer Lösung von Triaminopyridin in möglichst wenig verdünnter Salzsäure mit Platinchlorwasserstoffsäure entstand das Chloroplatinat, schöne, rotgelbe, verwachsene Nädelchen, die sich aber nach ganz kurzer Zeit schon in der Fällungsflüssigkeit dunkelrot färbten und sich an der Luft unter Platinabscheidung vollends zersetzten.

\section{Nachwort.}

Mein ausgezeichneter Mitarbeiter, Erich Ritter v. Beck, hat, nachdem er 10 Monate lang tapfer für das Vaterland gekämpft, am 10. Mai d. J. sein junges Leben lassen müssen. Mit ihm ist ein begabter Forscher, von dem die -Wissenschaft noch viele schöne Erfolge erwarten durfte, ins Grab gesunken. 